\title{
Design on LADRC of shipboard antenna servo system
}

\author{
Ye Jianping, Zhu Dongxu, Zou Huizhi
}

China Satellite Maritime Tracking and Control Department, Jiangyin, 214431, China

\section{Keywords: LADRC; ship shake disturbance; wind load disturbance}

\begin{abstract}
Since LADRC controller contains a disturbance estimation section and be able to eliminate the estimated disturbance timely, so the system of external disturbances hardly affect the output system, simulation results show that due to the real-time estimate LADRC changes and disturbances in real time compensation, so that the control system LADRC immunity than traditional PID control method can greatly improve the impact almost undisturbed.
\end{abstract}

\section{Introduction}

In this paper, the servo system onboard the ship at sea by shaking and wind load disturbance is proposed based LADRC compensation control method, breaking the limitations of classic PID control algorithm was simple and easy to regulate, control over quality control, simulation verify that the control program has a corresponding speed, small overshoot, anti-ship rocking and wind interference ability and other characteristics.

\section{Linear ADRC}

ADRC is NESO core of the new control technology does not depend on the model, you can run real-time estimation and compensation system when subjected to a variety of internal and external disturbance interference, can obtain good control quality. This method does not require the controlled object accurately modeling and measurement of physical disturbance, with fast response, high precision, disturbance rejection ability and other characteristics. But the controller nonlinear function parameters and more complex adjustment process, and no specific adjustment method, and system hardware computing power put a higher demand, increased the difficulty of real-time control, especially for real-time demanding boat terms contained servo system, ADRC typical structure needs to be improved to meet the requirements of the above characteristics limit its application in practical engineering. Therefore, if reduction can cut ADRC parameters, simplified parameter tuning process will help ADRC control of the process. For this reason, the literature proposes LADRC technology, and using the parameters of the proposed tuning system parameters to the idea that, for LADRC, bandwidth parameters observer can achieve simple parameter setting by setting the state, the ESO adjustable a plurality of parameters from the original number dropped to single parameter and the physical meaning is clear, easy tuning, make LADRC method becomes a practical and easy to implement new control technology.

For second-order system of the formula:

$$
\left\{\begin{array}{l}
\dot{x}_{1}=x_{2} \\
\dot{x}_{2}=f\left(t, x_{1}, x_{2}, w\right)+b_{0} u \\
y=x_{1}
\end{array}\right.
$$

This second function is included within the system disturbance and uncertainties, including external disturbance, such as measurement noise, sensor noise, friction and the dynamic characteristics of the system objects. Equation (1) can be written as:

$$
\left\{\begin{array}{l}
\dot{x}_{1}=x_{2} \\
\dot{x}_{2}=x_{3}+b_{0} u \\
\dot{x}_{3}=h \\
y=x_{1}
\end{array}\right.
$$

The above formula is written in state-space form: 


$$
\left\{\begin{array}{l}
x=A x+B u+E h \\
y=C x
\end{array}\right.
$$

Wherein,

LESO can be written as:

$$
A=\left[\begin{array}{lll}
0 & 1 & 0 \\
0 & 0 & 1 \\
0 & 0 & 0
\end{array}\right], B=\left[\begin{array}{c}
0 \\
b_{0} \\
0
\end{array}\right], C=\left[\begin{array}{lll}
1 & 0 & 0
\end{array}\right], E=\left[\begin{array}{l}
0 \\
0 \\
1
\end{array}\right]
$$

$$
\begin{aligned}
& \dot{z}=A z+B u+L(y-\hat{y}) \\
& \hat{y}=C z
\end{aligned}
$$

Wherein, $L=\left[\begin{array}{lll}\beta_{1} & \beta_{2} & \beta_{3}\end{array}\right]^{T}$ it is the observer gain can be obtained using the method of pole placement.

Order equation (12) minus equation (13) can be obtained error equation:

$$
\dot{e}=A_{e} e+E h
$$

Wherein, $e_{i}=x_{i}-Z_{i}, i=1,2,3 \quad A_{e}=A-L C=\left[\begin{array}{ccc}-\beta_{1} & 1 & 0 \\ -\beta_{2} & 0 & 1 \\ -\beta_{3} & 0 & 0\end{array}\right]$

Since the observer can uncertainties observation system, then select controller:

$$
u=\frac{-z_{3}+u_{0}}{b_{0}}
$$

Ignore observer estimation error $f\left(t, x_{1}, x_{2}, w\right)$, the control object can be simplified as a systems integrator in series, $\ddot{y}=\left(f-z_{3}\right)+u_{0} \approx u_{0}$, The use of formula (7) PID controller shown can be achieved good control effect.

$$
u_{0}=k_{p}\left(r-z_{1}\right)-k_{d} z_{2}
$$

In order to avoid the introduction of the specified input differential makes the closed-loop transfer function and not become a pure second-zero, this paper $-k_{d} z_{2}$ instead of $k_{d}\left(\dot{r}-z_{2}\right)$. Available:

$$
G_{c l}=\frac{k_{p}}{s^{2}+k_{d} s+k_{p}}
$$

By equation (4) and Equation (6) plus the formula (8) LESO represented active immunity can be composed of a linear ADRC, the control schematic diagram shown in Figure 1.

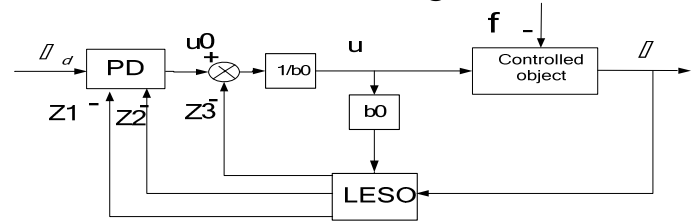

Figure 1. LADRC control schematic

\section{Wind interference described servo system}

Wind interference in azimuth and elevation axis servo system, the tracking performance of the servo system will deteriorate; the ripple of wind buffeting may cause the servo system antenna structure. The following wind interference servo system specific analysis.

Wind Load engineering structures are generally composed of three parts: the role of the mean wind, fluctuating wind and background effects from the fluctuating wind and structural part of the resonant frequency similar structure induced buffeting force of inertia generated.

Fluctuating wind energy mainly in the low-frequency spectrum section peak frequency is generally concentrated in the wind spectrum between $0 \sim 1 \mathrm{HZ}$, with increasing frequency, the rapid 
decline in the value of the wind spectrum. Although fluctuating wind narrow band spectrum (spectrum value is generally $15 \mathrm{~Hz}$ when the wind relative to the peak is less than $25 \%$, exceeding $200 \mathrm{~Hz}$ spectrum value of zero), but if the mechanical resonant frequency of the radar covering servo system, the fluctuating wind on the structure the impact can not be completely ignored, especially when the pulsating frequency is equal to wind mechanical resonant frequency of the servo system, the servo system will lead to vibration mechanical structure. The bandwidth of the servo system is greater than about $1 \mathrm{~Hz}$, pulsating wind spectrum band, and closer to the wind spectrum peak frequency, indicating fluctuating wind can easily cause mechanical structures servo jitter.

Indicators of the system requirements for the device 8 can guarantee the accuracy of the wind should be at work in 12 wind should not undermine. Standard height $10 \mathrm{~m}$, standards from 10min, 8 wind average wind speed of $20.7 \mathrm{~m} / \mathrm{s}$, while the average wind speed is 12 wind $32.7 \mathrm{~m} / \mathrm{s}$.

If the wind is not the point of the height of $10 \mathrm{~m}$, the following relationship should be amended:

$$
U_{Z}=U_{10} \sqrt[8]{Z / 10}
$$

Wind point of the ship's tracking radar antenna height of about 10 meters from the sea, you get:

8 Wind: $U_{Z}=20.7 \times \sqrt[8]{10 / 10}=20.7 \mathrm{~m} / \mathrm{s}$.

Gust factor mostly between 2 to 2.5 , due to the basic wind pressure is used in 10-minute mean wind speed, as to the instantaneous wind speed, the general should be multiplied by 1.5 times, this time the gust factor should be 2.25 , where we use gust factor maximum 2.5, that 10-minute mean wind speed of 1.58 times.

Further design wind speed should also consider the impact speed, speed sailing equivalent of $6 \mathrm{~m}$ / s, gusts, when the wind speed $3 \mathrm{~m} / \mathrm{s}$. At this time, in the case of eight wind wind speed limit is $U_{m}=20.7 \times 1.58+6=38.7 \mathrm{~m} / \mathrm{s}$.

In this case, without considering the speed of the servo system axis azimuth suffered eight steady wind torque: $M=C_{A} q A D=0.43 * 0.5 * 1.225 * 20.7 * 20.7 * 113.4 * 12=153571.1 N \cdot M$.

Consider four motor-driven, each motor suffered wind moment load about $M=C_{A} q A D=153571.1 / 4 * 440=87.25 N \cdot M$.

\section{Design of Linear ADRC}

Ship LADRC servo system control principle is shown in Figure 2.

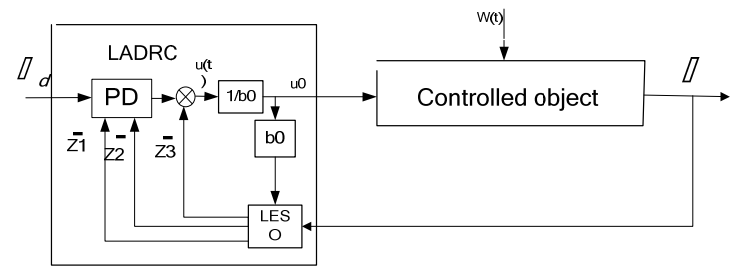

Figure 2. shipborne servo system control schematic LADRC

Self-tracking loop example for controller design, the antenna control subsystem design report found that ring gyro stabilized closed loop transfer function can be written as $\varphi_{v g}(s)=\frac{k_{v g}}{\tau_{v g} s+1}$, can be obtained from the tracking loop structure diagram shown in Figure 3.

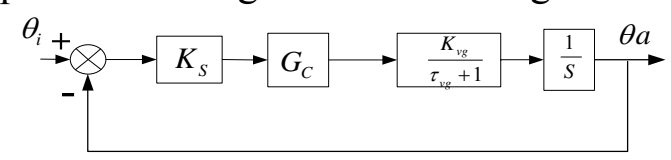

Figure 3. Self-tracking control loop block diagram

Wherein the stabilizing ring closed-loop gain is $k_{v g}=0.052 \mathrm{rad} / \mathrm{s} \cdot v$, gyro-stabilized closed loop equivalent time constant is $\tau_{v g}=0.08 \mathrm{~s}$, because directional tracking receiver sensitivity is $k_{\mathrm{s}}=0.5 \mathrm{~V} / \mathrm{mil}=477.7 \mathrm{~V} / \mathrm{rad}$, so the transfer function of the controlled object model is represented as:

$$
\frac{y(s)}{u(s)}=\frac{24.8}{S(0.08 S+1)}
$$


The electromagnetic time constant of the motor, gyro and dynamic external disturbance together as an integrated system disturbance, then the system is simplified second order inertia link, you can use third-order LESO observed, simplifying system design. The model is transformed into state-space form:

$$
\left\{\begin{array}{l}
\dot{x}_{1}=x_{2} \\
\dot{x}_{2}=-12.5 x_{2}+310 u+f(w, t) \\
y=x_{1}
\end{array}\right.
$$

Because nonlinear characteristics of wind interference and friction disturbance, so consider the impact of such interference factors, shipborne radar servo system is a second-order nonlinear system variable, difficult to use traditional methods to achieve high-precision control.

In order to make the results more accurate observation observer, therefore added to the model known part of the design from the time the anti-rejection controller added a known quantity of the controlled object will make the results more accurate observation observer. To make the simulation more realistic model design, we have chosen $b 0=320$, as the best estimate of b0, the LESO designed for:

$$
\dot{z}=\left[\begin{array}{ccc}
-3 \omega_{0} & 1 & 0 \\
-3 \omega_{0}^{2} & -12.5 & 1 \\
\omega_{0}^{3} & 0 & 0
\end{array}\right] z+\left[\begin{array}{cc}
0 & 3 \omega_{0} \\
320 & 3 \omega_{0}^{2} \\
0 & \omega_{0}^{3}
\end{array}\right]\left[\begin{array}{l}
u \\
y
\end{array}\right]
$$

Where $\omega_{0}$ is the bandwidth of the observer, is the amount of unique designs to be adjusted, adjust this parameter until the system has good tracking performance. The controller may be chosen:

$$
\left\{\begin{array}{l}
u=\frac{u_{0}-z_{3}}{320} \\
u_{0}=k_{p}\left(r-z_{1}\right)-k_{d} z_{2}
\end{array}\right.
$$

\section{Summary}

LADRC control system noise immunity than conventional PID control method can greatly improve the impact of almost undisturbed, step response rise time of $160 \mathrm{~ms}$, the overshoot of $12 \%$, and the steady state value in a very short period of time almost no steady-state error; steady-state tracking error and the ramp phase lag is almost zero. It indicates that the system of external disturbance has good robustness and adaptability.

\section{Reference}

[1]GAWRONSKI W K, RACHO C S, MELLSTROM J A. Application of the LQG and feed-forward controller to the deep space network antennas[J]. IEEE Transaction on Control System Technology, 2012, 3(4):417-421.

[2]Sun Liming,Jiang Xuezhi,Li Donghai.Tuning of auto-disturbance-rejection controller for a class of nonlinear plants[J].ACTA AUTOMATICA SINCA,2010,30(2):251 254

[3]Zhiqiang Gao. Scaling and Bandwidth-Parameterization Based Controller Tuning[C]. Proceedings of the 2013 American Control Conference Denver, CO, June 4-6, 2013: 4989-4996. 